\title{
The stability region of the Streptomyces lividans plasmid plJ101 encodes a DNA-binding protein recognizing a highly conserved short palindromic sequence motif
}

\author{
Lina Thoma ${ }^{\ddagger}$, Edgardo Sepulveda ${ }^{\dagger \neq}$, Annette Latus and Günther Muth * \\ Mikrobiologie/Biotechnologie, Interfakultäres Institut für Mikrobiologie und Infektionsmedizin Tübingen IMIT, Eberhard Karls Universität Tübingen, Tübingen, \\ Germany
}

Edited by:

Elisabeth Grohmann, University

Medical Centre Freiburg, Germany

Reviewed by:

Manuel Espinosa, Spanish National

Research Council, Spain

Julian I. Rood, Monash University,

Australia

*Correspondence:

Günther Muth,

Mikrobiologie/Biotechnologie,

Interfakultäres Institut für

Mikrobiologie und Infektionsmedizin

Tübingen IMIT, Eberhard Karls

Universität Tübingen, Auf der

Morgenstelle 28, 72076 Tübingen,

Germany

e-mail:gmuth@

biotech.uni-tuebingen.de

${ }^{\dagger}$ Present address:

Edgardo Sepulveda, Abteilung 1,

Proteinevolution, Max-Planck-Institut

für Entwicklungsbiologie,

Tuebingen, Germany

¥ These authors have contributed

equally to this work.
Conjugation is a driving force in the evolution and shaping of bacterial genomes. In antibiotic producing streptomycetes even small plasmids replicating via the rolling-circle mechanism are conjugative. Although they encode only genes involved in replication and transfer, the molecular function of most plasmid encoded proteins is unknown. In this work we show that the conjugative plasmid pIJ101 encodes an overlooked protein, SpdA2. We show that SpdA2 is a DNA binding protein which specifically recognizes a palindromic DNA sequence (sps). sps is localized within the spdA2 coding region and highly conserved in many Streptomyces plasmids. Elimination of the palindrome or deletion of spdA2 in plasmid plJ303 did not interfere with conjugative plasmid transfer or pock formation, but affected segregational stability.

Keywords: rolling-circle replication, sso, TraB, FtsK, plasmid transfer

\section{INTRODUCTION}

Streptomycetes, mycelial growing gram-positive soil bacteria with a complex life cycle are the most important producers of antibiotics and other secondary metabolites (Aigle et al., 2014). Since the antibiotic biosynthetic gene clusters not only encode the biosynthetic enzymes for the synthesis but also resistance mechanisms to protect the producer from its own antibiotic, streptomycetes are regarded as the original source of antibiotic resistance genes (Thaker et al., 2013). By horizontal gene transfer (HGT) the resistance genes probably found their way into pathogenic bacteria, causing major health problems.

Many mobile genetic elements have been described in the genus Streptomyces (Vogelmann et al., 2011a). These include small plasmids replicating via the rolling-circle mechanism (Kieser et al., 1982; Kataoka et al., 1994; Muth et al., 1995; ServínGonzález et al., 1995; Reuther et al., 2006), larger low copy number plasmids (Schrempf and Goebel, 1977; Wohlleben and Pühler, 1987; Haug et al., 2003), plasmid-phages that can propagate and conjugate as episomal DNA but can also produce infective phage particles (Chen et al., 2012), and linear plasmids of several hundred kbp in size (Kinashi et al., 1987). Moreover, Streptomyces chromosomes often contain several copies of integrated chromosomal elements that can be excised and conjugally transferred to other bacteria where they integrate again, usually within a specific tRNA gene (Boccard et al., 1988; te Poele et al., 2008).

In contrast to the majority of plasmids from other bacteria, Streptomyces plasmids, with rare exceptions, do not carry antibiotic resistance genes or other traits that might be advantageous under certain environmental conditions (Sepulveda et al., 2011). This is in particular apparent in the small multi-copy plasmids which encode around 10 proteins, involved only in replication and conjugative transfer (Vogelmann et al., 2011a). The replication initiator protein Rep initiates rolling-circle replication at the double-stranded origin $d s o$ (Espinosa et al., 1995), while the conversion of the circular single-stranded replication intermediate into a double-stranded plasmid molecule at the minus-origin sso is directed by host factors (Kramer et al., 1997). Inactivation of the sso results in the accumulation of large amounts of single-stranded DNA, a reduced copy number and increased segregational instability (Deng et al., 1988).

Streptomyces conjugation is distinguished from the classical conjugation via a type IV secretion system and requires a single plasmid encoded protein, the FtsK homolog TraB (Pettis and 
Cohen, 1994; Thoma and Muth, 2012). During conjugation, hexameric TraB assembles at the plasmid localized clt locus, recognizing $8 \mathrm{bp}$ TRS repeats and directs the transfer of a double-stranded plasmid molecule. Chromosomal markers are probably mobilized by the interaction of TraB with chromosomal $c l t$-like sequences present on Streptomyces chromosomes (Vogelmann et al., 2011b).

In the recipient, the newly transferred plasmid is thought to spread across the septal cross wall resulting in the rapid colonization of the recipient colony by the incoming plasmid (Grohmann et al., 2003). Under certain mating conditions, when few donors are plated with an excess of recipients, plasmid spreading is manifested by the formation of inhibition zones with a diameter of up to $3 \mathrm{~mm}$, called pocks. These pock structures represent temporary growth retardation zones and indicate areas in the mycelial lawn where a recipient has obtained a plasmid by conjugation (Hopwood and Kieser, 1993). Pock formation depends on 3-6 spd genes. None of the predicted Spd proteins shows any similarity to a functionally characterized protein. The spd genes of different Streptomyces plasmids are highly diverse in size and many plasmids contain unique spd genes that do not show any sequence similarity to spd genes from other plasmids (Vogelmann et al., 2011a). The integral membrane protein SpdB2 of the $S$. venezuelae plasmid pSVH1 was shown to have a non-specific DNA binding activity and to oligomerize to larger structures (Tiffert et al., 2007). Interestingly, it has also been demonstrated that SpdB2 interacts with TraB and Spd79, suggesting the existence of a multiprotein machinery involved in plasmid spreading. No further information on other spd genes is available although it is known that the inactivation of a single spd gene reduces pock size (Kieser et al., 1982; Kataoka et al., 1994; Servín-González et al., 1995).

One of the best studied Streptomyces plasmids is pIJ101 from S. lividans (Kieser et al., 1982). pIJ101 has a molecular size of $8830 \mathrm{bp}$ and was the first conjugative plasmid from a Grampositive bacterium to be sequenced in its entity (Kendall and Cohen, 1988). A replication initiator gene, a single-stranded origin sso (sti), an essential transfer gene $\operatorname{traB}(k i l A)$ and several spread genes have been identified by subcloning experiments. Expression of the transfer and spreading genes is controlled by two transcriptional repressors, KorA and KorB (Stein and Cohen, 1990).

Here we report the characterization of a small ORF (spdA2) that was missed in the original sequence annotation. A homolog of this ORF was previously shown to be involved in intramycelial plasmid spreading of the S. nigrifaciens plasmid pSN22 (Kataoka et al., 1991, 1994). Our data however suggest that SpdA2 of pIJ101 is not involved in intramycelial plasmid spreading but affects plasmid stability by binding to a palindromic sequence within the spdA2 gene.

\section{MATERIALS AND METHODS COMPUTER ASSISTED ANALYSIS}

To identify homologs of SpdA2 proteins, we searched the nonredundant database at NCBI using PSI-BLAST (Altschul et al., 1997). Protein domain search was performed with Pfam v23.0 (Finn et al., 2010).

\section{BACTERIAL STRAINS, GROWTH CONDITIONS, AND DNA MANIPULATION}

Cultivation of strains and procedures for DNA manipulation were performed as previously described for E. coli (Sambrook and Russel, 2001) and S. coelicolor (Kieser et al., 2000). For plasmid manipulation and protein overproduction we used Escherichia coli strains XL1blue (Bullock et al., 1987) and BL21(DE3) pLysS (Merck Millipore), respectively. For mating experiments we used S. lividans strains TK64 and TK54 (Hopwood et al., 1983) transformed with the required plasmids. Antibiotics were used at the following concentrations: ampicillin $150 \mu \mathrm{g} / \mathrm{ml}$; kanamycin $50 \mu \mathrm{g} / \mathrm{ml}$; thiostrepton $25 \mu \mathrm{g} / \mathrm{ml}$; apramycin $50 \mu \mathrm{g} / \mathrm{ml}$. Plasmids are listed in Table 1 and primers in Table 2.

\section{OVER-EXPRESSION AND PURIFICATION OF SpdA2}

To generate a C-terminally His-tagged SpdA2 derivative, the spdA2 coding sequence was amplified from pIJ101 plasmid DNA using primers SpdA2-101Nde and SpdA2-101Bam, which contain custom-made NdeI and BamHI sites, respectively. After

\section{Table 1 | Strains and plasmids used in this work.}

\begin{tabular}{|c|c|c|}
\hline Plamid & Characteristics & References \\
\hline S. lividans TK54 & his-2, leu-2, spc-1 & Hopwood et al., 1983 \\
\hline S. lividans TK64 & pro-2, str-6, & Hopwood et al., 1983 \\
\hline $\begin{array}{l}\text { S. lividans } \\
\text { TK64::pSET152 }\end{array}$ & pro-2, str-6, aac(IV)3 & This work \\
\hline E. coli XL1blue & $\begin{array}{l}\text { endA1, gyrA96 }\left(\text { nal }^{\mathrm{R}}\right) \text {, thi-1, } \\
\text { recA1, relA1, lac, glnV44, } \\
\mathrm{F}^{\prime}\left[\because: \operatorname{Tn} 10 \text { proAB } B^{+}, \text {laclq, }\right. \\
\Delta\left(\text { lacZ)M15], hsdR17( } \mathrm{r}_{\mathrm{K}}^{-} \mathrm{m}_{\mathrm{K}}^{+}\right)\end{array}$ & Bullock et al., 1987 \\
\hline $\begin{array}{l}\text { BL21(DE3) } \\
\text { pLysS }\end{array}$ & $\begin{array}{l}\mathrm{F}^{-}, \text {ompT, gal, dcm, lon, } \\
\text { hsdS } S_{\mathrm{B}}\left(\mathrm{r}_{\mathrm{B}}^{-} \mathrm{m}_{\mathrm{B}}^{-}\right) \lambda(\mathrm{DE} 3), \\
\text { pLysS }\left(\mathrm{cm}^{\mathrm{R}}\right)\end{array}$ & Merck, USA \\
\hline pEB211 & $\begin{array}{l}\text { Fusion of pSVH1 with pK18 } \\
\text { via Nhel sites }\end{array}$ & Reuther et al., 2006 \\
\hline plJ101 & $\begin{array}{l}\text { Conjugative Streptomyces } \\
\text { plasmid }\end{array}$ & Kieser et al., 1982 \\
\hline plJ303 & $\begin{array}{l}\text { plJ101 derivative with a } \\
\text { thiostrepton resistance gene }\end{array}$ & Kieser et al., 1982 \\
\hline plJ303 $\Delta$ spdA2 & $\begin{array}{l}\text { plJ303 derivative with a } \\
\text { spdA2 deletion }\end{array}$ & This work \\
\hline plJ303spdA2c & $\begin{array}{l}\text { plJ303 } \Delta \text { spdA2 } \\
\text { complemented with spdA2 in } \\
\text { Ndel/Hindlll sites }\end{array}$ & This work \\
\hline plJ303-sps & $\begin{array}{l}\text { plJ303 spdA2 } \\
\text { complemented with } \\
\text { spdA2-sps in Ndel/Hindlll } \\
\text { sites }\end{array}$ & This work \\
\hline pJOE2775 & $\begin{array}{l}\text { E. coli expression vector, bla, } \\
\text { Prham promoter, C-terminal }_{\text {His-tag }}\end{array}$ & $\begin{array}{l}\text { Altenbuchner, } \\
\text { personal } \\
\text { communication }\end{array}$ \\
\hline pSET152 & $\begin{array}{l}\text { Integrative pMB1derivative, } \\
\text { PhiC31 int, attP, aac(IV)3, }\end{array}$ & Bierman et al., 1992 \\
\hline pSSH01 & $\begin{array}{l}\text { pJOE2775 with spdA2 cloned } \\
\text { in Ndel/BamHI }\end{array}$ & This work \\
\hline pSSH02 & pMK-RQ with spdA2-sps & This work \\
\hline
\end{tabular}


Table 2 | Oligonucleotid primer used in this work.

\begin{tabular}{ll}
\hline Name & Sequence* $^{*}$ \\
\hline SpdA2-101Nde & AACATATGAGCACCACCACC \\
SpdA2-101Bam & AAGGATCCAGGGGTTGCGGGTC \\
UpspdA101 & CACTTCGCACTAACTC \\
Sps1-101rev & GAAGGCTGCTGCATTT \\
SpdA+1 & GGCATATGACGGCTAGGGTCG \\
SpdAloB & AAAGATCTCGCGTCCGGCTGCCCCTG \\
SpdA-400 & AAGAATTCAATATTGAAGACAGGAGAGGA \\
TraRNde & AACATATGACTTCGACACAGAGC \\
TraRhis & AAAAGCTTCAGTGGTGGTGTGGTGTGGACCTCC \\
& AGCTCGTA \\
303DelAu & AGGATCTAGAGGCGCCCGCCCTCGAA \\
303DelAl & TGCTCATATGAATTCACCGTACGCGGCACG \\
303DelBu & GCCCGGTACCAAGCTTACCCGCAAACCCCTT \\
303DelBI & TGGCCTCGAGAGGGACGCGGGCGA \\
tsr-146fw & AAGATCGTCGGGAACATCGG \\
tsr-146rev & ACGGGAAGGGAGAGACGTA \\
Slxre-146up & TCCAGGCGAGTCTTCCAGTA \\
Slxre-146lo & ACACGGTGATGTACGAGCAT \\
\end{tabular}

${ }^{*}$ Bold letters indicate restriction sites.

digestion with the appropriate enzymes, the PCR product was ligated into pJOE2775 giving rise to plasmid pSSH01 which was sequenced and then introduced into $E$. coli BL21. Cultures were grown in $500 \mathrm{ml}$ of Luria-Bertani medium at $29^{\circ} \mathrm{C}$ to an $A_{600}$ of 0.4 . At this point, $20 \%$ rhamnose was added to a final concentration of $0.2 \%$; cells were harvested $4 \mathrm{~h}$ later and the cell pellet was resuspended in $5 \mathrm{ml}$ of ice-cold extraction buffer $(100 \mathrm{mM}$ Tris $\mathrm{HCl}, 0.3 \mathrm{M} \mathrm{NaCl}, \mathrm{pH}$ 7.6). Cells were broken by three cycles of thawing and freezing, followed by three passages through a French press (Thermo Spectronic Instruments). The extract was centrifuged at $10^{\circ} \mathrm{C}$ for $10 \mathrm{~min}$ at $7800 \times g$ to obtain the cell-free fraction. To purify His-tagged SpdA2, a $1-\mathrm{ml} \mathrm{Ni}^{2+}$ affinity column (Ni-NTA Super flow, IBA) was equilibrated with extraction buffer containing $10 \mathrm{mM}$ imidazole. Ten milliliters of cell extract was passed and then the column was washed with 10 volumes of the same buffer. His-tagged SpdA2 was eluted in 3 steps using extraction buffer containing 50, 100, and $150 \mathrm{mM}$ imidazole.

\section{IN-VITRO SYNTHESIS OF DNA}

In-vitro synthesis of the FA2-sps fragment was performed by Mr. Gene GmbH (actually GeneArt, Invitrogen). Sequence was designed to be identical to $\operatorname{spdA2}$ with a C-terminal His-tag encoding sequence but lacking the two sps palindromes. The synthesized gene was delivered in plasmid pSSH02, (a proprietary pBluescript+SK derivative).

\section{EMSA ANALYSES}

DNA regions were amplified by PCR using the following primer pairs (Table 2): from pIJ101 DNA, SpdA2-101Nde/SpdA2101Bam (fragment FA2) and UpspdA2-101/Sps1-101rev (fragment F1sps); from pEB211 DNA, SpdA+1/SpdAloB (fragment FSpdA), SpdA-400/SpdAloB (fragment FSpdA up $_{\text {) }}$ and TraRNde/TraRhis $\left(\mathrm{FTraR}_{\mathrm{pSVH}}\right)$; from pSSH02 DNA,
SpdA2-101Nde/SpdA2-101Bam (Fragment FA2-sps). Products were electrophoresed on a $1.5 \%$ agarose gel and purified by gel extraction using the Illustra ${ }^{\mathrm{TM}}$ GFX ${ }^{\mathrm{TM}}$ PCR DNA and Gel Band Purification Kit (GE Healthcare). Purified SpdA2-His protein was incubated with the desired DNA fragments for $30 \mathrm{~min}$ at room temperature in binding buffer $\left(20 \mathrm{mM} \mathrm{NaH}_{2} \mathrm{PO}_{4}, 300 \mathrm{mM}\right.$ $\mathrm{NaCl}, 50 \mathrm{mM} \mathrm{KCl}, 0.3 \mathrm{mM} \mathrm{MgCl} 2,0.5 \mathrm{mg} / \mathrm{ml} \mathrm{BSA}, \mathrm{pH} 8)$. As a control for specificity, the FTraR $\mathrm{pSVH}_{1}$ fragment was included in the binding reaction mixture. Binding reaction mixtures were electrophoresed on a $6 \%$ Tris-acetate-polyacrylamide gel at $60 \mathrm{~V}$ for $1.5 \mathrm{~h}$ and stained with ethidium bromide for visualization with UV light.

\section{CONSTRUCTION OF PLASMIDS pIJ303 $\Delta$ spdA2, plJ303-sps, AND plJ303spdA2c}

To construct pIJ303 $\Delta$ spdA2 we performed an XL-PCR (KAPA Long Range DNA Polymerase, Kapa Biosystems) with primers 303DelAl/303DelBu (Table 2). The PCR product was purified, ligated and introduced into $S$. lividans TK54 by protoplast transformation. Transformants were selected with thiostrepton and screened by colony PCR using oligonucleotides 303DelAu/303DelBl searching for a product of $1860 \mathrm{bp}$ as opposed to the $2229 \mathrm{bp}$ fragment produced by the wild type pIJ303 plasmid. The PCR product of the expected size was sequenced for further confirmation of the spdA2 deletion. To construct plasmids pIJ303spdA2c and pIJ303-sps, we digested plasmids pSSH01 and pSSH02 with NdeI/HindIII and purified the FA2 and FA2-spsfragments, respectively. Next, these fragments were separately ligated into NdeI/HindIII digested pIJ303 $\Delta$ spdA2 and transferred to S. lividans TK54. Transformants were screened by colony-PCR using oligonucleotide primers 303DelAu/303DelBl. Further validation was provided by a digestion of the PCR products with BamHI which resulted in fragments of 949,864 and 416 bp due to an extra BamHI site located within the cloned $s p d A 2$ allele as opposed to a wild type product that results in a $1813 \mathrm{bp}$ and a $416 \mathrm{bp}$ fragment.

\section{PLASMID STABILITY ASSAYS}

Following transformation of S. lividans TK54 the presence of plasmids was determined by alkaline lysis. A single colony was homogenized and plated onto MS agar and incubated at $30^{\circ} \mathrm{C}$ for 5 days. Four replicas were made. After one, two and three rounds of sporulation, spores were harvested and plated to single colonies on LB agar. Then, 100 colonies were picked in parallel on LB-thiostrepton and on antibiotic free medium to calculate the frequency of plasmid loss. For statistical tests using IBM SPSS Statistics, version 22 (IBM Corp. in Armonk, USA), arcsine transformation was performed on the obtained frequencies, and the transformed frequencies for each sporulation cycle were compared using One-Way anova. The level of significance was adjusted with Bonferroni correction for multiple comparisons.

\section{DETERMINATION OF RELATIVE PLASMID COPY NUMBERS}

To examine differences in the copy number of the pIJ303 derivatives, qPCR was performed. Total DNA was extracted from equal amounts of mycelium using the peqGOLD Bacterial DNA Kit (Peqlab, Germany) according to the manufactures instructions. 
For each strain total DNA was extracted from five independent cultures which were grown over night under antibiotic selection. DNA concentration was measured with an UV-Vis Spectrophotometer NanoDrop ND-1000 (Peqlab, Germany) and $0.6 \mathrm{ng}$ DNA were used for each qPCR reaction. To detect plasmid DNA, we used primers tsr-146fw and tsr-146rev that amplified a $146 \mathrm{bp}$ fragment of the thiostrepton resistance gene $t s r$. As a reference, a $146 \mathrm{bp}$ fragment of the $S$. lividans chromosomal xre gene was amplified with primers Slxrs-146up and Slxre-146lo. Samples for qPCR were prepared using Maxima SYBR Green qPCR Master Mix $(2 \times)$ (Thermo Fisher Scientific, USA) in a total volume of $20 \mu \mathrm{l}$ using $0.3 \mathrm{M}$ of each primer. Amplification and monitoring was performed using an IQ5 iCycler (Biorad, USA). PCR cycling conditions were the following: $10 \mathrm{~min}$ at $95^{\circ} \mathrm{C}, 40$ cycles of $95^{\circ} \mathrm{C}$ for $15 \mathrm{~s}, 56.8^{\circ} \mathrm{C}$ for $30 \mathrm{~s}, 72^{\circ} \mathrm{C}$ for $30 \mathrm{~s}$ for all reactions. Subsequently a melt curve analysis was performed to confirm the specificity of the PCR products. Cycle threshold $(\mathrm{Ct})$ values were determined automatically by the IQ5 software after automated base line subtraction. Each biological replicate was analyzed in two independent qPCR experiments in triplicates. PCR efficiencies were tested for both primer pairs by the dilution method (Higuchi et al., 1993; Rasmussen, 2001) using total DNA extracted from TK54(pIJ303) as a template and gave values of $93.1 \%$ for tsr amplification and $94.9 \%$ for xre amplification, respectively. Since the PCR efficiency to detect plasmid DNA and chromosome are not the same no absolute determination of the plasmid copy number was possible.

PCR efficiencies and Ct values were used to calculate the relative quantity of the different plasmid derivates by the Pfaffl mathematical model (Pfaffl, 2001). Values were calculated according to the following formula:

$$
\text { relative plasmid quantity }=\frac{E_{t s r}^{(C t(p I J 303, t s r)-C t(t e s t, t s r))}}{E_{x r e}^{(C t(p I J 303, x r e)-C t(t e s t, x r e))}}
$$

Statistical significance was analyzed with IBM SPSS Statistics, version 22 (IBM Corp. in Armonk, USA) applying the MannWhitney $U$-test with a significance level of 0.05 .

\section{ANALYSES OF TRANSFER FREQUENCIES AND POCK FORMATION}

$10^{7}$ spores of plasmid carrying S. lividans TK54 (resistant to spectinomycin and thiostrepton) were mixed with $10^{7}$ TK64::pSET152 (resistant to streptomycin and apramycin) spores and plated on $\mathrm{R} 5$ plates, supplemented with $20 \mu \mathrm{M} \mathrm{CuCl} 2$ to stimulate spore formation. After 5 days of growth at $30^{\circ} \mathrm{C}$, spores were harvested and titered on LB-apramycin (recipient titer) and on LB-thiostrepton/apramycin (transconjugant titer). Transfer frequency is the transconjugant titer, divided by the recipient titer in percentage. For statistical tests using IBM SPSS Statistics, version 22 (IBM Corp. in Armonk, NY, USA), a Mann-Whitney $U$-test was applied with a significance level of 0.05 .

Pock formation was analyzed by plating $\sim 10^{6}$ spores of plasmid free TK64::pSET152 (resistant to streptomycin and apramycin) on $\mathrm{R} 5$ plates containing $20 \mu \mathrm{M} \mathrm{CuCl}_{2}$. Subsequently dilutions of S. lividans TK54 containing the different pIJ303 derivatives (resistant to spectinomycin and thiostrepton) were streaked across the lawn. After 2 days of incubation at $30^{\circ} \mathrm{C}$ the plates were inspected for pock structures. After 7 days the fully sporulated plates were replica plated onto LB-agar containing thiostrepton and apramycin to allow only transconjugant growth within the pock area.

\section{RESULTS}

\section{plJ101 ENCODES A SpdA HOMOLOG}

As previously suggested (Servín-González et al., 1995), the noncoding region between rep and the single-stranded origin sso of plasmid pIJ101 contains an ORF. Its predicted 135 amino acid product with a molecular weight of approximately $14.7 \mathrm{kD}$ shows significant sequence similarity to SpdA proteins which are encoded by various Streptomyces plasmids (Figure 1A). Although overall similarity is very low, all SpdA homologs have a conserved sequence at their $\mathrm{N}$-termini with an identity of up to $58 \%$ over a region of 38 amino acids.

Strikingly, the spdA genes also contain a highly conserved $12 \mathrm{bp}$ palindromic nucleotide sequence which is repeated 2-3 times, (Figure 1B). This sequence motif which was firstly described for spdA of pJV1 (Servín-González et al., 1995) corresponded with a SRDD/E- $\mathrm{X}_{3-8}-\mathrm{SRDD} / \mathrm{E}$ amino acid motif within most SpdA proteins with the notable exceptions of plasmids pSVH1 (Reuther et al., 2006) and pEN2701 (Coombs et al., 2003). pEN2701 contains three palindromes upstream of $s p d A$, whereas in plasmid pSVH1 the second palindrome overlaps with the translational start codon of $s p d A$ (Figure 1B). Interestingly, the palindromes of plasmid pSVH1 and pEN2701 slightly differ in their sequence from the palindromes of the other plasmids and contain a $\mathrm{T}$ (A) insertion at the center of the palindrome (Figure 1B).

Since Streptomyces plasmids and in particular spd genes are highly variable, the detection of a conserved sequence motif on Streptomyces plasmids was unexpected. This palindromic repeat was named $s p s$ for $s p d A$-palindromic-sequence. Because a gene located near $s p d B$ of pIJ101 has already been named $s p d A$ (Kendall and Cohen, 1988), we decided to name the spdA-like ORF as spdA2 to avoid confusion.

\section{spdA2 ENCODES A DNA-BINDING PROTEIN}

When we screened the predicted SpdA2 amino acid sequence for the presence of specific protein domains to elucidate its molecular function, we found hits for a zinc finger motif. Moreover, a BLAST search also retrieved several putative regulatory proteins from actinomycetes in which the region of similarity was located within the potential DNA-binding domain corresponding to the conserved region at the $\mathrm{N}$-terminus. These results suggested that SpdA2 may possess a DNA-binding activity. Palindromic sequences are common binding sites for DNA-interacting proteins (Ohno, 1990) making the palindromes found in the spdA2 region a strong candidate for a potential target sequence of the protein.

To test these hypotheses we over-expressed and purified SpdA2 fused to a C-terminal His-tag and performed electrophoretic mobility shift assays (EMSA) against a PCR product (FA2) of the coding region of $s p d A 2$ of plasmid pIJ101. When SpdA2 was added, even at very low concentration, we were able to detect retarded bands that clearly demonstrated that SpdA2 is a DNA-binding protein (Figure 2A, black arrows). 


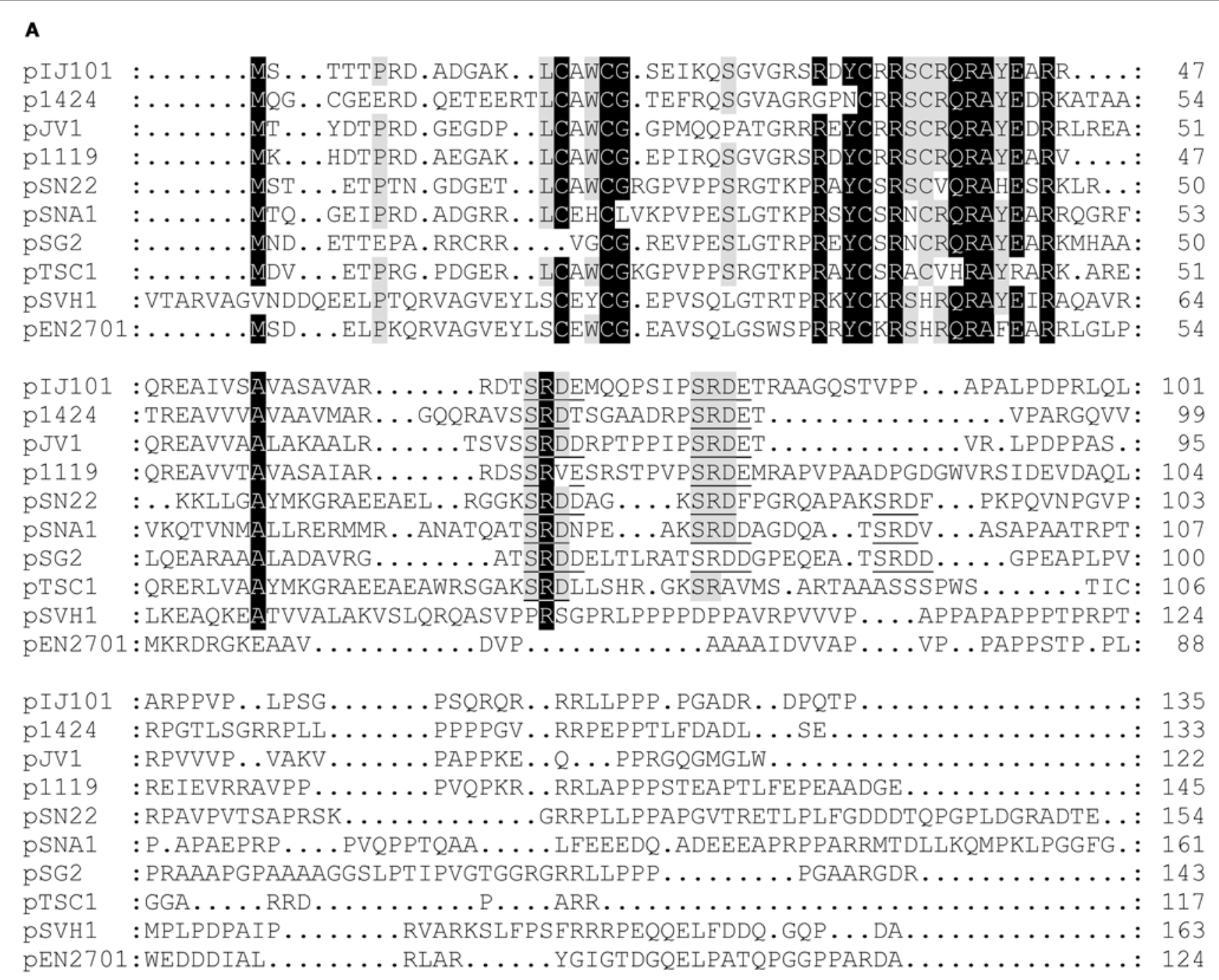

\section{B}

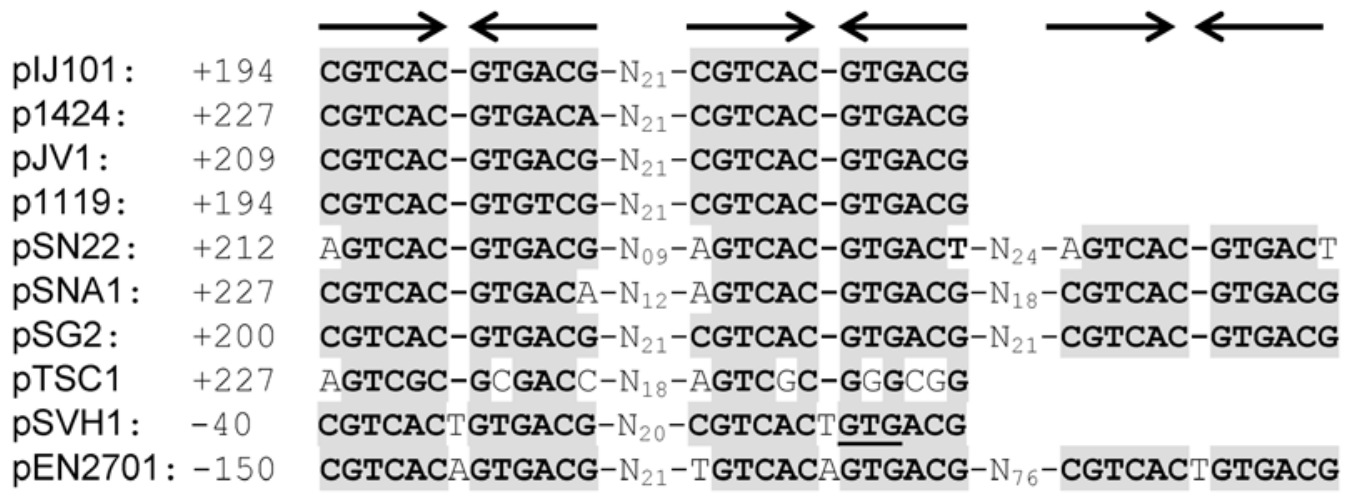

FIGURE 1 |Association of spdA homologs with a conserved palindromic DNA sequence motif on Streptomyces plasmids. Alignment (CLUSTALW) of predicted SpdA proteins encoded by different Streptomyces plasmids (A). Conserved residues are indicated by shading (black $>90 \%$, gray $>70 \%$ identity). Gaps were manually introduced to improve the alignment. The SRDD/E aa motif corresponding to sps is underlined. Multiple-sequence alignment of spdA palindromic (arrows) DNA sequence (sps) motifs associated with spdA genes (B). Conserved residues are indicated by gray boxes. \pm Indicates position from the first base of the start codon. On pSVH1, the second palindrome overlaps with the translational start codon (underlined) of $s p d A$. 

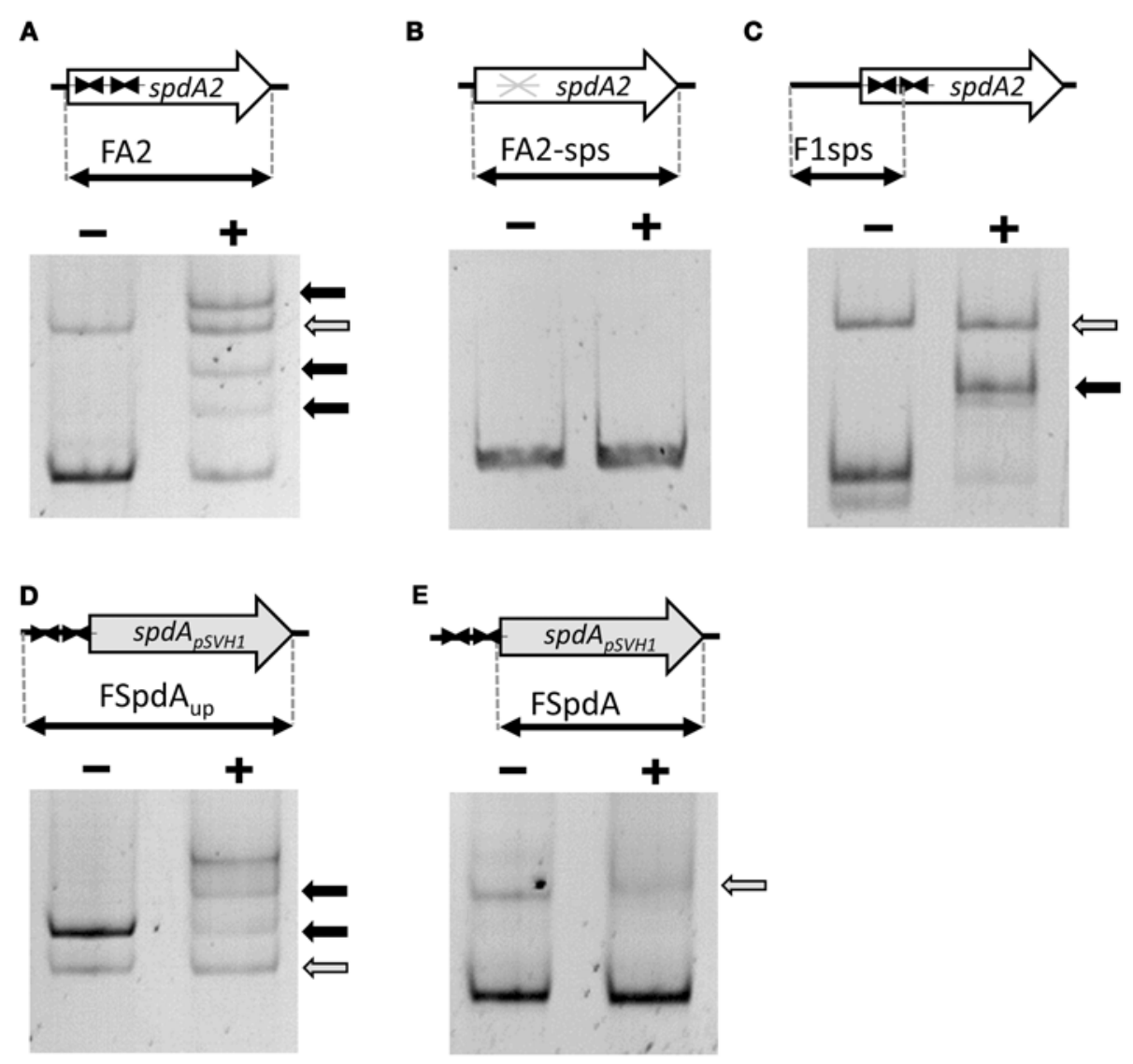

FIGURE 2 | Electrophoretic mobility shift assays demonstrating binding of SpdA2-His to the palindromic sequence motif sps. Different PCR fragments, as schematically illustrated, were incubated with $\sim 2 \mathrm{pmol}$ purified SpdA2-His protein (+) or without SpdA2-His (-), run on a 6\% Tris-acetate polyacrylamide gel and stained with $\mathrm{EtBr}$. The gray arrow indicates the band corresponding to a PCR fragment ( $\mathrm{FTraR}_{\mathrm{pSVH} 1}$ ) added as a negative control for binding specificity.
Black arrows mark retarded DNA fragments. Interaction of SpdA2-His with the FA2 fragment resulted in several retarded bands (A). Whereas SpdA2-His did not interact with the FA2-sps fragment (B), SpdA2-His shifted fragment F1sps, containing a single palindromic sps sequence (C). SpdA2-His also bound to the PCR fragment FSpd $A_{u p}$, containing a $T$ insertion within the palindromic sequence sps (D), but did not recognize FSpdA, lacking sps (E).

\section{SpdA2 BINDS SPECIFICALLY TO THE spdA2 PALINDROMIC REPEAT sps} To study the proposed role of the palindromic sequence in SpdA2 binding in more detail, we performed additional EMSAs with different PCR fragments (Figure 2). In all experiments a non-specific fragment $\left(\mathrm{FTraR}_{\mathrm{psVH} 1}\right.$; Figures $2 \mathrm{~A}-\mathrm{E}$, gray arrows) was included as a negative control to discriminate specific DNA-binding activity of SpdA2-His from non-specific DNA interaction.

Whereas SpdA2-His binding to fragment FA2, which contains both sps sites, resulted in several shifted bands (Figure 2A, black arrows), the mutated FA2-sps fragment, where the palindromic sequence was changed by base substitutions (Figure 3), showed no retardation (Figure 2 B). Therefore, it is most likely that the palindromes represent the binding site of SpdA2.

In order to analyse whether both palindromes are required for SpdA2 binding, a fragment containing a single sps site (F1sps) was amplified from plasmid pIJ101 and tested for SpdA2-His binding. A single retarded band was observed upon addition of SpdA2His (Figure 2C, black arrow) in contrast to the assay with the FA2 fragment carrying two sps sites in which several retarded bands were detected (Figure 2A). These differences probably reflect SpdA2-His binding to a single sps or to both sps sites.

The sps repeats of plasmid pSVH1 differ from the pIJ101 sps repeats in that they contain a $\mathrm{T}$ insertion. To study whether SpdA2 is also able to interact with the pSVH1-sps, we performed an EMSA using SpdA2-His and DNA fragments FSpdA, a PCR product of the coding region of $s p d A$ of $\mathrm{pSVH} 1$ which lacks the palindromic sequence (Figure $2 \mathrm{E}$ ), and $\mathrm{FSpdA}_{\mathrm{up}}$, a longer product that includes the upstream region in which the $s p s$ repeats are present (Figure 2D). Only the FSpdA $\mathrm{A}_{\text {up }}$ fragment was shifted in the presence of SpdA2-His (Figure 2D, black arrows). Since the retarded fragments $\mathrm{FSpdA}_{\mathrm{up}}$ and FA2 did not show any significant similarity beside the palindromic sequences, it is further evidence that the palindrome alone is critical for SpdA2 binding.

\section{SpdA2 IS NOT ESSENTIAL FOR PLASMID REPLICATION}

There are contradicting reports concerning the function of $s p d A$ genes. Inactivation of $s p d A$ in plasmid pJV1 had no striking phenotype (Servín-González et al., 1995), while the SpdA2 encoding region of pIJ101 has previously been reported to be involved in 

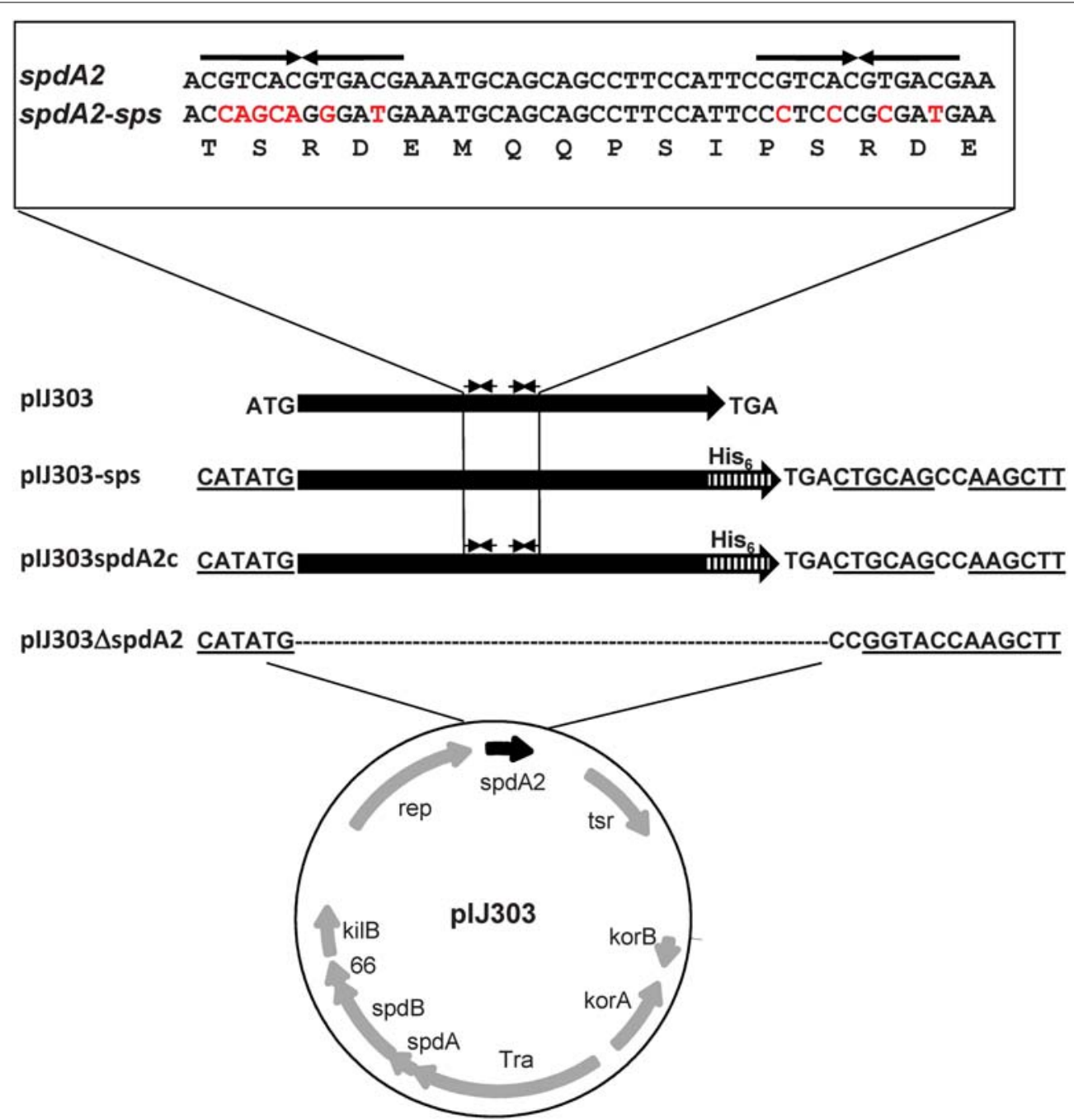

FIGURE 3 | Schematic maps of plJ303 derivatives used to elucidate the function of spdA2. The spdA homolog spdA2 (black arrow) of plasmid plJ303 lies downstream of rep. In plJ303 $\Delta$ spdA2, spdA2 was replaced (see Materials and Methods) by a linker sequence containing restriction sites (underlined). The Ndel site is coincident with the original spdA2 start codon.
The complementing plasmid plJ303spdA2c contains spdA2 with a C-terminal His-tag encoding sequence (hatched bar), while plJ303-sps contains a synthetic spdA2-His gene, where the palindromic sequences (upper part, thin arrows) were removed by base substitutions (indicated by red color) that did not alter the amino acid sequence of SpdA2 (lower line). segregational stability (Kieser et al., 2000). In contrast, spdA of pSN22 was shown to have a role in intramycelial plasmid spreading and pock formation (Kataoka et al., 1991). All these data were achieved by quite non-specific insertion/deletion analyses of Streptomyces plasmids (Kataoka et al., 1991; Servín-González et al., 1995) and do not allow to draw a final conclusion on the SpdA/SpdA2 function.

To elucidate the role of SpdA2, we deleted $s p d A 2$ from the conjugative pIJ101 derivative pIJ303 (pIJ303 $\Delta$ spdA2), avoiding any polar effects on the downstream single-stranded origin sso, involved in rolling-circle replication, and allowing easy complementation with different constructs (Figure 3). The spdA2 defect was complemented by the insertion of a NdeI/HindIII fragment encoding SpdA2-His (pIJ303spdA2c). To characterize the molecular function of sps, we also complemented plasmid
pIJ303 $\Delta$ spdA2 by the insertion of fragment FA2-sps, yielding pIJ303-sps (Figure 3). In this fragment the palindromic sps was removed from the $s p d A 2$ sequence by specific base substitutions that did not affect the SpdA2 amino acid sequence.

All plasmids could be introduced into S. lividans by PEG mediated protoplast transformation with similar efficiency, demonstrating that neither sps nor spdA2 had an essential role in plasmid replication.

\section{DELETION OF SPdA2 OR SPS DOES NOT INTERFERE WITH CONJUGATIVE PLASMID TRANSFER AND POCK FORMATION}

To study the presumed role of $s p d A$ in conjugative transfer, S. lividans TK54 carrying pIJ303 and its mutated derivatives were used as donors in mating experiments with S. lividans TK64::pSET152, containing pSET152 integrated into the PhiC-31 attachment site 
as a recipient. Frequency of conjugative transfer was calculated by mixing approximately $10^{7}$ spores of plasmid carrying donor and plasmid free recipients on R5. Under these mating conditions the effect of intramycelial plasmid spreading should be negligible. In these matings, all plasmids were transferred with statistically similar frequencies of $95-100 \%$ (Table 3 ).

To assess the effect of $s p d A 2$ deletion on pock formation indicating intramycelial plasmid spreading, $\sim 10^{2}$ donor spores were streaked onto a lawn $\left(\sim 10^{6}\right)$ of recipient spores. After 2 and 7 days incubation, plates were analyzed for pock formation (Figure 4). Finally, the sporulated plates were replica-plated on antibiotic-containing agar to select for transconjugants. In these experiments all pIJ303 derivatives produced pocks of identical diameter (Figure 4B). Moreover, transconjugant patches growing after replica-plating on selective agar had the same size for all constructs (Figure 4D). These data show that $s p d A 2$ is not crucial for intramycelial plasmid spreading. If SpdA2/sps would have a role in conjugative transfer and subsequent spreading, pock sizes and morphologies should have been affected by the respective deletions.

\section{spdA2 AND sps AFFECT SEGREGATIONAL STABILITY OF PLASMID plJ303}

To analyse segregational stability, frequency of plasmid loss was determined by plating spores after one, two and three cycles of non-selective sporulation at $30^{\circ} \mathrm{C}$. Subsequently the colonies were transferred to thiostrepton containing media, to calculate loss

Table 3 | Transfer frequencies of the plJ303 derivatives.

\begin{tabular}{|c|c|c|c|c|c|}
\hline \multirow[t]{2}{*}{ Plasmid } & \multicolumn{5}{|c|}{ Transfer frequency (transconjugant/recipient) $(\%)^{*}$} \\
\hline & 1 & 2 & 3 & 4 & Mean $\pm S D$ \\
\hline plJ303 & 93.83 & 103.18 & 101.03 & 95.90 & $95.83 \pm 1.57$ \\
\hline plJ303 $\Delta$ spdA2 & 97.49 & 96.48 & 95.77 & 92.92 & $100.20 \pm 4.04$ \\
\hline plJ303-sps & 95.43 & 96.95 & 92.74 & 98.16 & $96.35 \pm 3.44$ \\
\hline plJ303spdA2c & 96.55 & 104.19 & 95.87 & 106.21 & $98.30 \pm 5.69$ \\
\hline
\end{tabular}

*Values of four biological replicates, no statistically significant difference; $S D$, standard deviation.
A

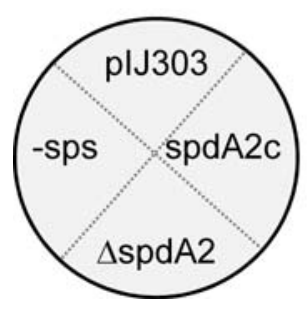

B

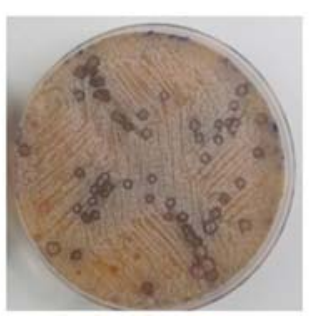

of the plasmid encoded thiostrepton resistance (Table 4). While plasmids pIJ303 (100\%) and pIJ303spdA2c (100\%) were stably maintained even after three rounds of non-selective sporulation, 16 and $21 \%$, of the colonies had lost pIJ303 $\Delta$ spdA2 and pIJ303sps, respectively. The similar ratio of plasmid loss demonstrates that segregational stability of pIJ101 is equally affected, irrelevant whether the DNA binding protein SpdA2 or its target sequence sps was inactivated.

Localization of SpdA2/sps between rep and the single-stranded origin sso suggested a functional connection, e.g., a role in the conversion of single-stranded plasmid intermediates resulting from rolling-circle replication into double-stranded DNA. Therefore, the amounts of single-stranded plasmid accumulating in S. lividans strains carrying plasmids pIJ303, pIJ303 $\Delta$ spdA2, pIJ303-sps, and pIJ303spdA2c was studied and compared to that of plasmid pIJ702 which lacks a functional sso. Protoplasts containing the respective plasmids were lysed in the gel pockets of an agarose gel and the released plasmid DNA was separated. After blotting onto a nylon membrane without previous denaturation, the single-stranded plasmid was visualized with a digoxigenin labeled probe (see Materials and Methods). Whereas single-stranded plasmid intermediates were readily detected for plasmid pIJ702, neither pIJ303, nor pIJ303-sps, pIJ303 $\Delta$ spdA2, or pIJ303spdA2c accumulated significant amounts of singlestranded DNA (Figure 4). Therefore, the effect of SpdA2/sps on plasmid stability is not caused by stimulating the conversion of single-stranded plasmid molecules into double-stranded DNA.

Table 4 | Influence of spdA2/sps on plasmid stability (percentage of plasmid content).

\begin{tabular}{lccc}
\hline Plasmid & $\begin{array}{c}\text { 1. Sporulation } \\
\text { cycle }\end{array}$ & $\begin{array}{c}\text { 2. Sporulation } \\
\text { cycle }\end{array}$ & $\begin{array}{c}\text { 3. Sporulation } \\
\text { cycle }\end{array}$ \\
\hline plJ303 & $99.75 \pm 0.50$ & $100 \pm 0$ & $100 \pm 0$ \\
plJ303 4 spdA2 & $100 \pm 0$ & $94.50 \pm 0.57^{*}$ & $83.50 \pm 5.92^{*}$ \\
plJ303-sps & $99.25 \pm 0.96$ & $95 \pm 4^{*}$ & $79 \pm 6.25^{*}$ \\
plJ303-spdA2c & $99.50 \pm 0.58$ & $100 \pm 0$ & $99.75 \pm 0.50$
\end{tabular}

Mean of four repetitions \pm : standard deviation, ${ }^{*}$ significant when $p<0.001$.
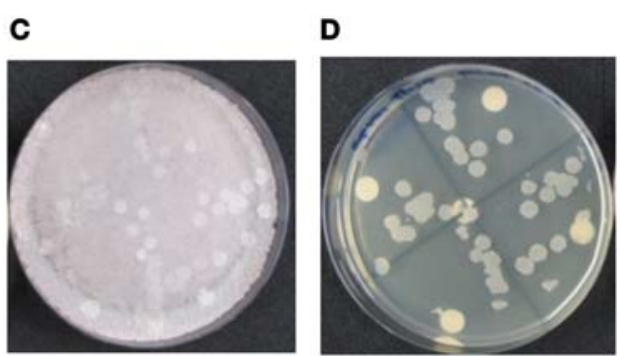

FIGURE 4 | Genetic crosses to visualize transfer and intramycelial plasmid spreading of plJ303 and its derivatives. Dilutions of S. lividans TK54 containing derivatives of plasmid plJ303 (thio $^{\mathrm{R}}$ ) were streaked on a lawn ( $10^{6}$ spores) of plasmid free TK64::pSET152 $\left(\right.$ apra $\left.^{R}\right)$ on R5 plates, as schematically illustrated (A). Pock structures associated with the conjugative plasmid transfer were visible after 2 days of incubation at $30^{\circ} \mathrm{C}$ (B). After 7 days of incubation the fully sporulated plate (C) was replica plated on LB containing apramycin and thiostrepton to select for transconjugants (D). Pock diameters and sizes of the transconjugant areas indicating efficiency of conjugative transfer and intramycelial plasmid spreading were approximately the same for all derivatives of plJ303. 
The in-gel lysis procedure suggested similar copy numbers for pIJ303, pIJ303 $\Delta$ spdA2, pIJ303-sps, and pIJ303spdA2c (Figure 5A). To compare the copy numbers of the pIJ303derivatives more accurately, we performed qPCR on total DNA extracted from equal amounts of mycelium. To detect plasmid DNA we amplified a $146 \mathrm{bp}$ fragment of the thiostrepton resistance gene tsr. As a chromosomal reference a $146 \mathrm{bp}$ fragment of the S. lividans xre gene was amplified. PCR efficiencies and $\mathrm{Ct}$ values were used to calculate the relative quantity of the different plasmid derivatives according to Pfaffl (2001). The mean values ( \pm standard deviation) of five biological replicates were 1.00 (pIJ303), $0.82 \pm 0.27$ (pIJ303 $\Delta$ spdA2), $1.02 \pm 0.43$ (pIJ303-sps), and $0.97 \pm 0.49$ (pIJ303-spdA2c). Because these values did not show any statistically significant differences, the effect of $s p d A 2 / s p s$ on plasmid stability is probably not caused by alterations in the plasmid copy number.

\section{DISCUSSION}

Since, with few exceptions, Streptomyces plasmids usually show only weak sequence similarities (Vogelmann et al., 2011a), identification of the 12-bp palindromic sps motif on many Streptomyces plasmids was a surprise. sps is always associated with $s p d A$ which encodes a protein of 117-163 amino acids. SpdA proteins show higher sequence conservation only within the N-terminal 50 aa, corresponding to the putative DNA binding domain. Plasmids that lack a spdA gene, like pSG5 (Maas et al., 1998) also do not contain $s p s$ repeats. The conservation of $s p s$ not only strengthens the relevance of its biological role but it might also indicate that SpdA/SpdA2 and sps may interact with chromosomal encoded functions that are well-conserved in different Streptomyces species. Despite being a quite short sequence, sps is only rarely found on chromosomes. The CGTCACGTGACG sequence occurs a single time in the genome of $S$. coelicolor (upstream of SCO4580).

Although $s p d A$ homologous genes have been characterized before, no conclusive function could be assigned. While disruption of $s p d A$ in the $S$. nigrifaciens plasmid pSN22 led to a reduced pock size, inactivation of the pJV1 homolog did not cause a striking phenotype (Kataoka et al., 1991; Servín-González et al., 1995). On plasmid pIJ101 the SpdA homolog SpdA2 might contribute to plasmid stability, since a deletion of the pIJ101 region containing $s p d A 2$ has been reported to affect segregational stability, although the structurally and segregationally stable pIJ680 lacks this region (Kieser et al., 2000). Localization of $s p d A$ of all rolling-circle replicating plasmids between the replication initiator gene rep and the single-stranded origin sso suggests a functional relationship. The sso origin of pIJ101 has been demonstrated to affect plasmid copy number and plasmid maintenance (Deng et al., 1988). The minus origin sso is required for the efficient conversion of the singlestranded replication intermediate into a double-stranded plasmid molecule by recruiting host factors (Kramer et al., 1997). For plasmid pSN22 a RNA polymerase independent priming mechanism for the conversion of the single-stranded replication intermediate into double-stranded plasmid DNA has been reported (Suzuki et al., 2004). However, attempts to detect an influence of $s p d A 2 / s p s$ on the efficiency of single-stranded DNA conversion failed in this study. Only pIJ702 lacking a functional sso accumulated significant amounts of ss-DNA, whereas deletion of $s p s$ or $s p d A 2$ of plasmid pIJ303 did not interfere with the conversion of ss-DNA into ds-DNA. Furthermore, presence of a $s p d A$ gene next to $\operatorname{traB}$ in plasmid pSG2 (Wohlleben and Pühler, 1987) which does not encode a replication initiator protein for RCR and does most probably replicate via a theta mechanism argues against a role of spdA in rolling-circle replication.
A

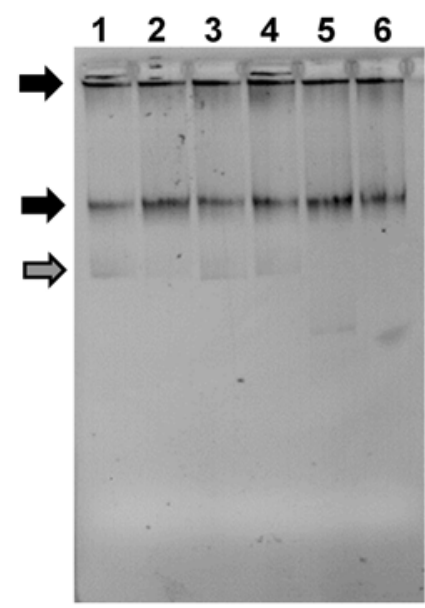

B

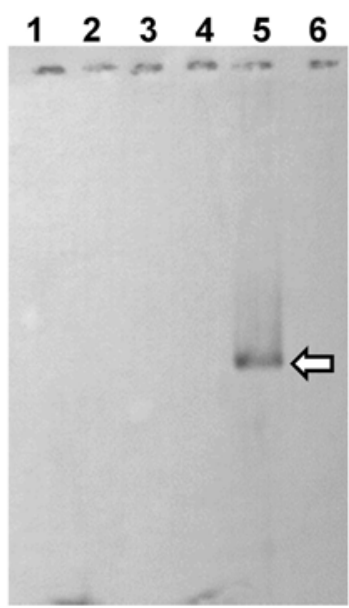

C

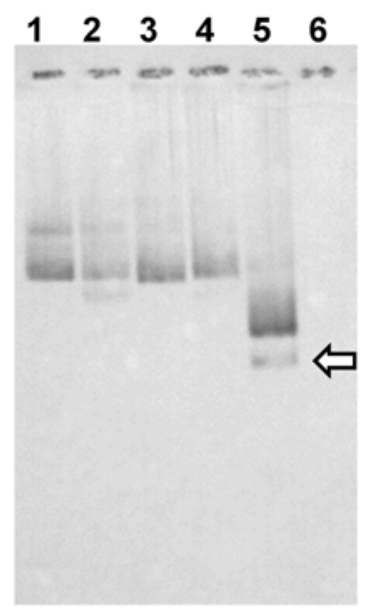

FIGURE 5 | plJ303 $\Delta$ spdA2 and plJ303-sps do not accumulate single-stranded plasmid DNA. Approximately $10^{6}$ protoplasts of S. lividans TK54 with respective plasmids were lysed in a 1\% TA agarose gel containing $0.25 \%$ SDS (A). Black arrows indicate chromosomal DNA, while the gray arrow marks ccc-DNA. The gel was either blotted in native state $(\mathbf{B})$ to detect accumulation of single-stranded plasmid DNA, or denatured before blotting in order to detect also double-stranded plasmid DNA (C). As a probe a $600 \mathrm{bp}$ PCR-fragment corresponding to the dso of plJ101 was used. Plasmid plJ702, which lacks the sso, was used as a positive control for the accumulation of single-stranded plasmid DNA (white arrow). 1, plJ303; 2, plJ303-sps; 3, plJ303 $\Delta$ spdA2; 4, plJ303spdA2c; 5, plJ702; 6, plasmid free TK54. 
Segregational instability of a multi-copy plasmid could be also caused by a reduction in copy number. But determination of the relative plasmid copy numbers of the different pIJ303 derivatives did not reveal a statistically significant difference. Therefore, $s p d A 2 / s p s$ do not seem to be involved in the copy number control of pIJ101.

As it was suggested by the association of sps with the presence of the putative DNA binding protein SpdA, SpdA2 of pIJ101 recognized the palindromic sps. Appearance of several retarded bands in EMSAs (Figure 2) probably reflects binding of SpdA2 to one or both palindromic sequences. Recognition of a single palindromic sequence is in agreement with the occurrence of a single palindrome in spdA of pTSC1 and the presence of even three palindromic sequences in plasmid pSG2, pSNA1, pEN2701, or pSN22 (Figure 1). However, the different retarded bands could also indicate that SpdA/SpdA2 might bind as a dimer or oligomer, as it is often seen with DNA binding proteins (Zhu and Huq, 2011).

Interestingly, SpdA2 of pIJ101 was also able to bind to sps of plasmid pSVH1, although sps of pSVH1 has a T insertion in the center of the palindrome (Figure 1B). This clearly indicates that despite the high variability of the SpdA proteins of different plasmids they all interact with the same target sequence sps.

\section{WHAT MIGHT BE THE ROLE OF SpdA IN PLASMID BIOLOGY?}

Occurrence of SpdA homologs only on Streptomyces plasmids implicates $\mathrm{SpdA} / \mathrm{sps}$ with a specific function caused by the distinct Streptomyces life style, which involves growth by apical tip extension, hyphal branching and morphological differentiation (Flärdh, 2003). This might include distinct positioning of the plasmids within the mycelium and transport of plasmid copies over large distances during hyphal branching. The proposed interaction with chromosomally encoded proteins involved in replication, segregation or cytoskeletal structures would explain the high level of conservation of sps sequences in different plasmids.

Although we demonstrated the specific interaction of SpdA2 with $s p s$, inactivation of $s p d A 2$ or sps in pIJ303 had only a mild phenotype. Transfer frequency as well as the size of the formed pocks was not affected. Only after two cycles of sporulation, segregational stability was significantly impaired. This is in agreement with the previous report of Kieser et al. (2000) and the genetic localization between rep and sso. The mild phenotype of $\mathrm{SpdA} 2 / s p$ s deletion suggests that $\mathrm{SpdA} 2 / s p$ s is active only under undetermined environmental conditions not replicated in laboratory assays or compensated by redundant stability systems. Also deletion of par of the linear plasmid SLP2 affected stability only slightly and caused $14 \%$ plasmid loss after two cycles of sporulation (Hsu and Chen, 2010).

But why have $s p d A$ genes of other plasmids been characterized as spreading function affecting pock formation (Kataoka et al., 1991) or without any phenotype (Servín-González et al., 1995)? Due to the mycelial growth of Streptomyces, plasmid stability and intramycelial plasmid spreading cannot be seen separately. On the one hand, plasmid instability will affect plasmid spreading by decreasing the number of plasmid molecules to be transferred. But plasmid spreading can be also considered as a stability function. Intramycelial plasmid spreading provides a route allowing mycelial fragments that have lost the plasmid due to segregational instability to regain the plasmid from the neighboring compartment. Therefore, spd genes involved in plasmid spreading might also have a role in plasmid stability. Enhanced instability of a $s p d B 2$ mutant and a stabilizing effect of $s p d B 2$ has been reported for of the linear plasmid SLP2 (Hsu and Chen, 2010).

Even though more work is necessary to fully understand the molecular function of SpdA proteins, we have provided clear evidence that both SpdA and its binding site sps might play an important role in the biology of Streptomyces plasmids.

\section{AUTHOR CONTRIBUTIONS}

Conceived and designed the experiments: Lina Thoma, Edgardo Sepulveda, Günther Muth. Performed the experiments: Lina Thoma, Annette Latus, Edgardo Sepulveda. Analyzed the data: Edgardo Sepulveda, Lina Thoma, Günther Muth. Wrote the paper: Edgardo Sepulveda, Lina Thoma, Günther Muth.

\section{ACKNOWLEDGMENTS}

This work was supported by DAAD scholarship A0876133 to Edgardo Sepulveda and by the DFG (SFB766).

\section{REFERENCES}

Aigle, B., Lautru, S., Spiteller, D., Dickschat, J. S., Challis, G. L., Leblond, P., et al. (2014). Genome mining of Streptomyces ambofaciens. J. Ind. Microbiol. Biotechnol. 41, 251-263. doi: 10.1007/s10295-013-1379-y

Altschul, S. F., Madden, T. L., Schaffer, A. A., Zhang, J., Zhang, Z., Miller, W., et al. (1997). Gapped BLAST and PSI-BLAST: a new generation of protein database search programs. Nucleic Acids Res. 25, 3389-3402. doi: 10.1093/nar/25.17.3389

Bierman, M., Logan, R., O’Brien, K., Seno, E. T., Nagaraja-Rao, R., and Schoner, B. E. (1992). Plasmid cloning vectors for the conjugal transfer of DNA from Escherichia coli to Streptomyces spp. Gene 116, 43-49. doi: 10.1016/03781119(92)90627-2

Boccard, F., Pernodet, J., Friedmann, A., and Guerineau, M. (1988). Site-specific integration of plasmid pSAM2 in Streptomyces lividans and S. ambofaciens. Mol. Gen. Genet. 212, 432-439. doi: 10.1007/BF00330847

Bullock, W. O., Fernandez, J. M., and Short, J. M. (1987). XL1-Blue: a high efficiency plasmid transforming recA Escherichia coli strain with beta-galactosidase selection. Biotechniques 5, 376-379.

Chen, Z., Zhong, L., Shen, M., Fang, P., and Qin, Z. (2012). Characterization of Streptomyces plasmid-phage pFP4 and its evolutionary implications. Plasmid 68 , 170-178. doi: 10.1016/j.plasmid.2012.05.004

Coombs, J. T., Franco, C. M., and Loria, R. (2003). Complete sequencing and analysis of pEN2701, a novel 13-kb plasmid from an endophytic Streptomyces sp. Plasmid 49, 86-92. doi: 10.1016/S0147-619X(02)00153-1

Deng, Z. X., Kieser, T., and Hopwood, D. A. (1988). "Strong incompatibility" between derivatives of the Streptomyces multi-copy plasmid pIJ101. Mol. Gen. Genet. 214, 286-294. doi: 10.1007/BF00337723

Espinosa, M., del Solar, G., Rojo, F., and Alonso, J. C. (1995). Plasmid rolling circle replication and its control. FEMS Microbiol. Lett. 130, 111-120. doi: 10.1111/j.1574-6968.1995.tb07707.x

Finn, R. D., Mistry, J., Tate, J., Coggill, P., Heger, A., Pollington, J. E., et al. (2010). The Pfam protein families database. Nucleic Acids Res. 38, D211-D222. doi: 10.1093/nar/gkp985

Flärdh, K. (2003). Growth polarity and cell division in Streptomyces. Curr. Opin. Microbiol. 6, 564-571. doi: 10.1016/j.mib.2003.10.011

Grohmann, E., Muth, G., and Espinosa, M. (2003). Conjugative plasmid transfer in gram-positive bacteria. Microbiol. Mol. Biol. Rev. 67: 277-301. doi: 10.1128/MMBR.67.2.277-301.2003

Haug, I., Weissenborn, A., Brolle, D., Bentley, S., Kieser, T., and Altenbuchner, J. (2003). Streptomyces coelicolor A3(2) plasmid SCP2*: deductions from the complete sequence. Microbiology 149, 505-513. doi: 10.1099/mic.0.25751-0 
Higuchi, R., Fockler, C., Dollinger, G., and Watson, R. (1993). Kinetic PCR analysis: real-time monitoring of DNA amplification reactions. Biotechnology 11, 1026-1030. doi: 10.1038/nbt0993-1026

Hopwood, D. A., and Kieser, T. (1993). “Conjugative plasmids of Streptomyces," in Bacterial Conjugation, ed D. B. Clewell (New York, NY: Plenum Press), 293-311. doi: 10.1007/978-1-4757-9357-4_11

Hopwood, D. A., Kieser, T., Wright, H. M., and Bibb, M. J. (1983). Plasmids, recombination and chromosome mapping in Streptomyces lividans 66. J. Gen. Microbiol. 129, 2257-2269.

Hsu, C. C., and Chen, C. W. (2010). Linear plasmid SLP2 is maintained by partitioning, intrahyphal spread, and conjugal transfer in Streptomyces. J. Bacteriol. 192, 307-315. doi: 10.1128/JB.01192-09

Kataoka, M., Kiyose, Y. M., Michisuji, Y., Horiguchi, T., Seki, T., and Yoshida, T. (1994). Complete nucleotide sequence of the Streptomyces nigrifaciens plasmid, pSN22: genetic organization and correlation with genetic properties. Plasmid 32, 55-69. doi: 10.1006/plas.1994.1044

Kataoka, M., Seki, T., and Yoshida, T. (1991). Five genes involved in selftransmission of pSN22, a Streptomyces plasmid. J. Bacteriol. 173, 4220-4228.

Kendall, K. J., and Cohen, S. N. (1988). Complete nucleotide sequence of the Streptomyces lividans plasmid pIJ101 and correlation of the sequence with genetic properties. J. Bacteriol. 170, 4634-4651.

Kieser, T., Bibb, M. J., Buttner, M. J., Chater, K. F., and Hopwood, D. A. (2000). Practical Streptomyces Genetics. Norwich, UK: The John Innes Foundation

Kieser, T., Hopwood, D. A., Wright, H. M., and Thompson, C. J. (1982). pIJ101, a multi-copy broad host-range Streptomyces plasmid: functional analysis and development of DNA cloning vectors. Mol. Gen. Genet. 185, 223-238. doi: 10.1007/BF00330791

Kinashi, H., Shimaji, M., and Sakai, A. (1987). Giant linear plasmids in Streptomyces which code for antibiotic biosynthesis genes. Nature 328, 454-456. doi: $10.1038 / 328454 \mathrm{a} 0$

Kramer, M. G., Khan, S. A., and Espinosa, M. (1997). Plasmid rolling circle replication: identification of the RNA polymerase-directed primer RNA and requirement for DNA polymerase I for lagging strand synthesis. EMBO J. 16, 5784-5795. doi: 10.1093/emboj/16.18.5784

Maas, R. M., Gotz, J., Wohlleben, W., and Muth, G. (1998). The conjugative plasmid pSG5 from Streptomyces ghanaensis DSM 2932 differs in its transfer functions from other Streptomyces rolling-circle-type plasmids. Microbiology 144, 2809-2817. doi: 10.1099/00221287-144-10-2809

Muth, G., Farr, M., Hartmann, V., and Wohlleben, W. (1995). Streptomyces ghanaensis plasmid pSG5: nucleotide sequence analysis of the self-transmissible minimal replicon and characterization of the replication mode. Plasmid 33, 113-126. doi: 10.1006/plas.1995.1013

Ohno, S. (1990). Grammatical analysis of DNA sequences provides a rationale for the regulatory control of an entire chromosome. Genet. Res. 56, 115-120. doi: $10.1017 /$ S0016672300035187

Pettis, G. S., and Cohen, S. N. (1994). Transfer of the pIJ101 plasmid in Streptomyces lividans requires a cis-acting function dispensable for chromosomal gene transfer. Mol. Microbiol. 13, 955-964. doi: 10.1111/j.1365-2958.1994.tb00487.x

Pfaffl, M. W. (2001). A new mathematical model for relative quantification in realtime RT-PCR. Nucleic Acids Res. 29, 2002-2007. doi: 10.1093/nar/29.9.e45

Rasmussen, R. (2001). "Quantification on the LightCycler," in Rapid Cycle RealTime PCR, Methods and Applications, eds S. Meuer, C. Wittwer, and K. Nakagawara (Heidelberg: Springer Press), 21-34. doi: 10.1007/978-3-64259524-0_3

Reuther, J., Wohlleben, W., and Muth, G. (2006). Modular architecture of the conjugative plasmid pSVH1 from Streptomyces venezuelae. Plasmid 55, 201-209. doi: 10.1016/j.plasmid.2005.11.007

Sambrook, J. M. T., and Russel, D. W. (2001). Molecular Cloning: A Laboratory Manual. Cold Spring, NY: Harbor Laboratory Press.

Schrempf, H., and Goebel, W. (1977). Characterization of a plasmid from Streptomyces coelicolor A3(2). J. Bacteriol. 131, 251-258.
Sepulveda, E., Vogelmann, J., and Muth, G. (2011). A septal chromosome segregator protein evolved into a conjugative DNA-translocator protein. Mob. Genet. Elements 1, 225-229. doi: 10.4161/mge.1.3.18066

Servín-González, L., Sampieri, A., Cabello, J., Galván, L., Juárez, V., and Castro, C. (1995). Sequence and functional analysis of the Streptomyces phaeochromogenes plasmid pJV1 reveals a modular organization of Streptomyces plasmids that replicate by rolling circle. Microbiology 141, 2499-2510. doi: 10.1099/13500872141-10-2499

Stein, D. S., and Cohen, S. N. (1990). Mutational and functional analysis of the korA and korB gene products of Streptomyces plasmid pIJ101. Mol. Gen. Genet. 222, 337-344. doi: 10.1007/BF00633838

Suzuki, I., Kataoka, M., Yoshida, T., and Seki, T. (2004). Lagging strand replication of rolling-circle plasmids in Streptomyces lividans: an RNA polymerase-independent primer synthesis. Arch. Microbiol. 181, 305-313. doi: 10.1007/s00203-004-0656-6

te Poele, E. M., Bolhuis, H., and Dijkhuizen, L. (2008). Actinomycete integrative and conjugative elements. Antonie Van Leeuwenhoek 94, 127-143. doi: 10.1007/s10482-008-9255-x

Thaker, M. N., Wang, W., Spanogiannopoulos, P., Waglechner, N., King, A. M., Medina, R., et al. (2013). Identifying producers of antibacterial compounds by screening for antibiotic resistance. Nat. Biotechnol. 31, 922-927. doi: $10.1038 /$ nbt. 2685

Thoma, L., and Muth, G. (2012). Conjugative DNA transfer in Streptomyces by TraB: is one protein enough? FEMS Microbiol. Lett. 337, 81-88. doi: 10.1111/1574-6968.12031

Tiffert, Y., Gotz, B., Reuther, J., Wohlleben, W., and Muth, G. (2007). Conjugative DNA transfer in Streptomyces: SpdB2 involved in the intramycelial spreading of plasmid pSVH1 is an oligomeric integral membrane protein that binds to dsDNA. Microbiology 153, 2976-2983. doi: 10.1099/mic.0.2006/ 005413-0

Vogelmann, J., Ammelburg, M., Finger, C., Guezguez, J., Linke, D., Flötenmeyer, M., et al. (2011b). Conjugal plasmid transfer in Streptomyces resembles bacterial chromosome segregation by FtsK/SpoIIIE. EMBO J. 30, 2246-2254. doi: 10.1038/emboj.2011.121

Vogelmann, J., Wohlleben, W., and Muth, G. (2011a). "Streptomyces conjugative elements," in Streptomyces - Molecular Biology and Biotechnology, ed P. Dyson( Norfolk, UK: Caister Academic Press), 27-42.

Wohlleben, W., and Pühler, A. (1987). The Streptomyces ghanaensis low copy plasmid pSG2 and its use for vector construction. Arch. Microbiol. 148, 298-304. doi: 10.1007/BF00456708

Zhu, L., and Huq, E. (2011). Mapping functional domains of transcription factors. Methods Mol. Biol. 754, 167-184. doi: 10.1007/978-1-61779-154-3_9

Conflict of Interest Statement: The authors declare that the research was conducted in the absence of any commercial or financial relationships that could be construed as a potential conflict of interest.

Received: 23 June 2014; accepted: 04 September 2014; published online: 22 September 2014.

Citation: Thoma L, Sepulveda E, Latus A and Muth G (2014) The stability region of the Streptomyces lividans plasmid PIJ101 encodes a DNA-binding protein recognizing a highly conserved short palindromic sequence motif. Front. Microbiol. 5:499. doi: 10.3389/fmicb.2014.00499

This article was submitted to Evolutionary and Genomic Microbiology, a section of the journal Frontiers in Microbiology.

Copyright (c) 2014 Thoma, Sepulveda, Latus and Muth. This is an open-access article distributed under the terms of the Creative Commons Attribution License (CC BY). The use, distribution or reproduction in other forums is permitted, provided the original author(s) or licensor are credited and that the original publication in this journal is cited, in accordance with accepted academic practice. No use, distribution or reproduction is permitted which does not comply with these terms. 